\title{
VAPOUR ABSORPTION IN MELAMINE-FORMALDEHYDE RESIN
}

\author{
J. Wawryszczuk, K. Ciesielski \\ Institute of Physics, Maria Curie-Skłodowska University \\ Pl. M. Curie-Skłodowskiej 1, 20-031 Lublin, Poland \\ AND J. GowOREK \\ Faculty of Chemistry, Maria Curie-Skłodowska University \\ Pl. M. Curie-Skłodowskiej 3, 20-031 Lublin, Poland
}

\begin{abstract}
The effects of vapour absorption on positron annihilation in cross-linked polymer were investigated. Contrary to linear polymers, absorption of vapour in cross-linked resin leads to the shortening of $o$-Ps lifetime. In vacuum absorbed molecules diffuse out. At room temperature desorption of hexane and hexanol is not complete; full elimination of absorbed molecules requires sample temperature increase.

PACS numbers: 78.70.Bj, 36.10.Dr, 61.41.+e, 66.30.-h
\end{abstract}

\section{Introduction}

Penetration of vapours and gases into the polymer structure is important from the viewpoint of practical application, e.g.:

- Porous rigid resins are in common use as column packing in chromatography, however, in most cases organic polymers swell in contact with vapour (or liquid) changing their pore characteristics. It can be seen in Fig. 1, where the pore distribution in melamine-formaldehyde resin (MFR) for dry and water saturated sample are presented;

- Resins are used as encapsulating materials for integrated circuits, protecting them from moisture, dust, shock etc. Diffusion of water molecules into resin affects its protective properties.

In absorption vapour molecules fill small voids in the polymer structure or distort the chain geometry. Changes in the size and number of free volume holes can be studied using the positron lifetime technique. In a series of papers Ito et al. [1-4] investigated positronium lifetime and intensity variation mainly for linear polymers absorbing water, hexane, benzene etc. In this paper we want to present some results concerning the influence of vapour absorption and desorption on the positron lifetime in non-porous cross-linked MFR. 


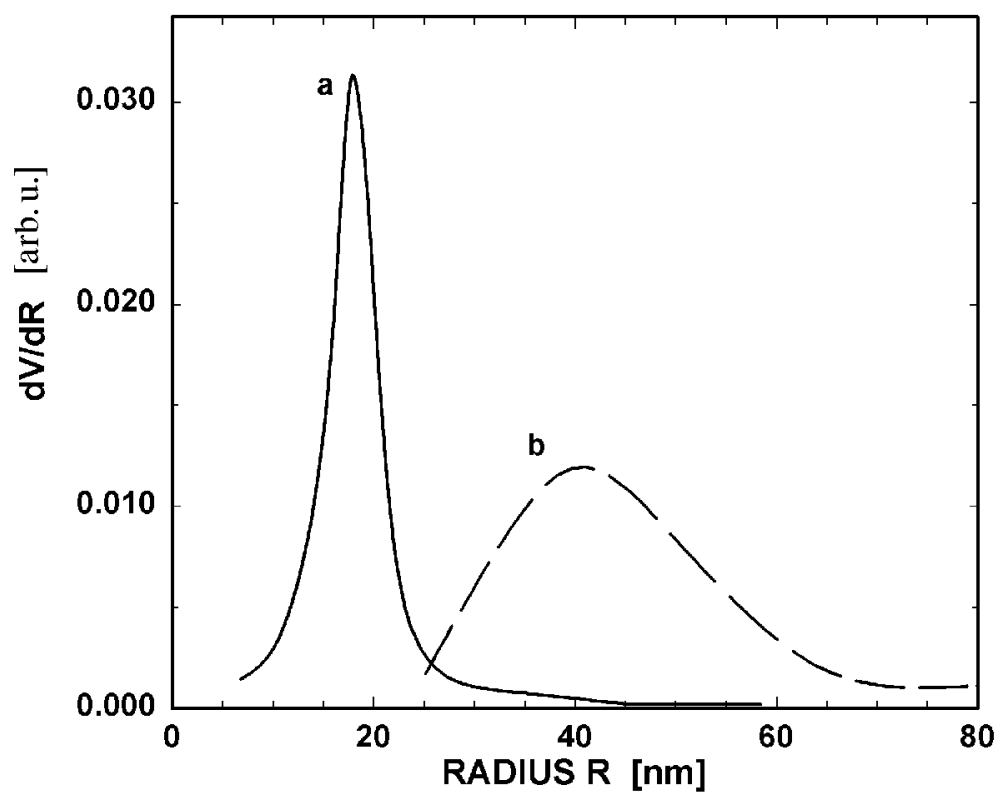

Fig. 1. Distribution of pore radii in porous melamine-formaldehyde resin: $a$ - dry sample, $b$ - wetted sample.

\section{Experimental}

The resin was prepared by mixing melamine, para-formaldehyde and polymerization initiator, aging it during 3 days and heating to $450 \mathrm{~K}$ for $3 \mathrm{~h}$. Fine grained (diameter $2-8 \mu \mathrm{m}$ ) resin showed the specific surface of $\approx 6 \mathrm{~m}^{2}$, thus negligible. Two layers of grains with positron source between them were placed in a small cylindrical container fixed in vacuum chamber and viewed by two scintillation counters. The positron lifetime spectra were registered using a standard fast-slow delayed coincidence spectrometer with 210 ps resolution, FWHM, and analysed by POSITRONFIT program [5], assuming 3 exponential components. The long-lived component with the lifetime $\tau_{3}$ and intensity $I_{3}$ was ascribed to the decay of ortho-positronium in small voids in the polymer structure. No very long-lived component ( $\tau>10 \mathrm{~ns}$ ) characteristic of $o$-Ps in pores was found.

The chamber contained an arm with a valve, behind which an ampoule with liquid could be placed. The chamber was evacuated to the pressure $\approx 0.3 \mathrm{~Pa}$ and then the valve was opened allowing the vapour to fill the whole chamber volume. Immediately after valve opening lifetime measurements started; a series of measurements was continued up to stabilization of the spectrum parameters; the time of one spectrum collection was $3 \mathrm{~h}$.

\section{Results and discussion}

The lifetime spectra of positrons annihilating in the raw ("as produced") sample were first measured as a function of temperature. The lifetime of the component belonging to $o$-Ps is shown in Fig. 2. The sample, initially kept at $95 \mathrm{~K}$, 
was heated up to $330 \mathrm{~K}$, stored $15 \mathrm{~h}$ at that temperature and next the sequence of temperatures was repeated and extended up to $450 \mathrm{~K}$. In the second run the values of lifetime and intensity were slightly higher than those in the first one. After the second series and $15 \mathrm{~h}$ storage at $450 \mathrm{~K}$ the sample was sufficiently aged and further runs with rising and decreasing temperature gave the $\tau_{3}$ and $I_{3}$ values changing along the same curve; both parameters were fully reproducible for the same MFR sample. Change of the sample to another one resulted in a slight modification of parameters, but the shape of temperature dependence remained the same as in Fig. 2 . Above $450 \mathrm{~K}$ the $\tau_{3}$ rose faster with temperature. This was expected because the glass transition point for our MFR polymer was located near $450 \mathrm{~K}$. The lifetime of $o-\mathrm{Ps}$ is rather short; $\tau_{3} \approx 1.4 \mathrm{~ns}$ corresponds, according to Tao-Eldrup model [6], to the radius of free volume hole $R=0.225 \mathrm{~nm}$.

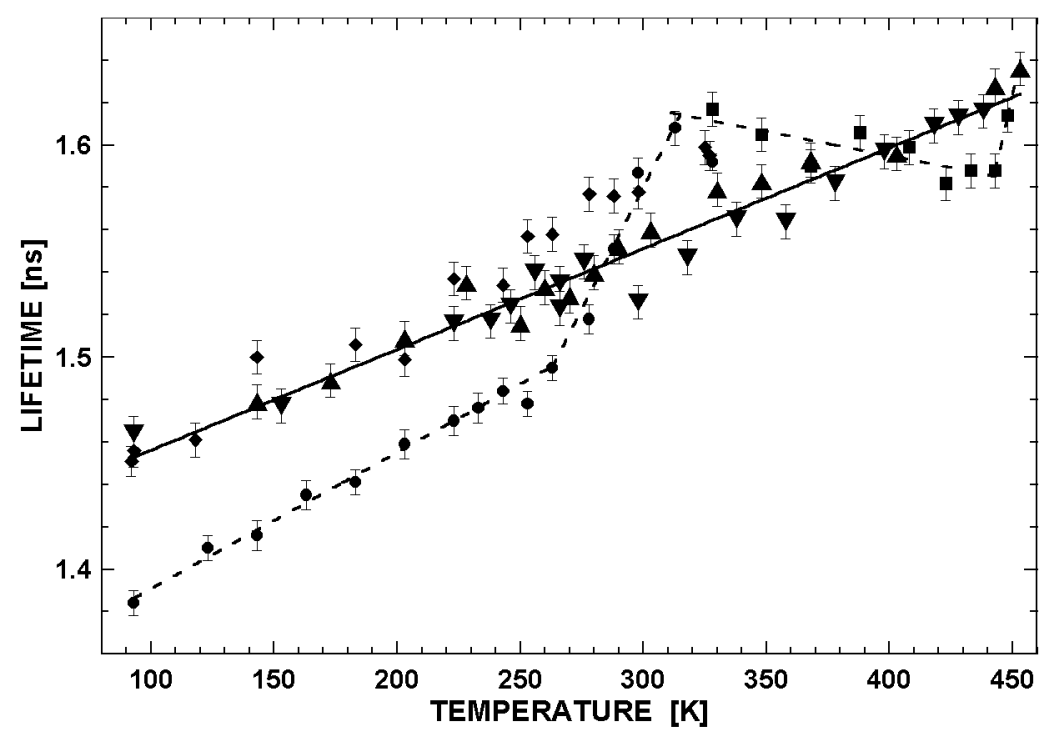

Fig. 2. Temperature dependence of $o$-Ps lifetime $\tau_{3}$ in MFR. Dots and dashed line first run; diamonds and squares - second run; triangles — next runs (straight line is fitted to the data from these runs). Triangles denote the series with rising temperature, inverted triangles — with decreasing temperature.

Measurements of absorption effects were performed at room temperature, i.e. in glassy state of MFR. The changes of $o$-Ps lifetime as a function of time of resin exposure on vapour are shown in Fig. 3. Filling the sample chamber with dry air has practically no influence on $\tau_{3}$. In the case of water, hexane, and hexanol one observes shortening of the lifetime with progressing absorption; exposure on benzene vapour gives no measurable effect. Particularly effective is water absorption; $\tau_{3}$ shortening in the presence of hexane vapour is 10 times slower despite a much higher hexane vapour pressure than that of water. 


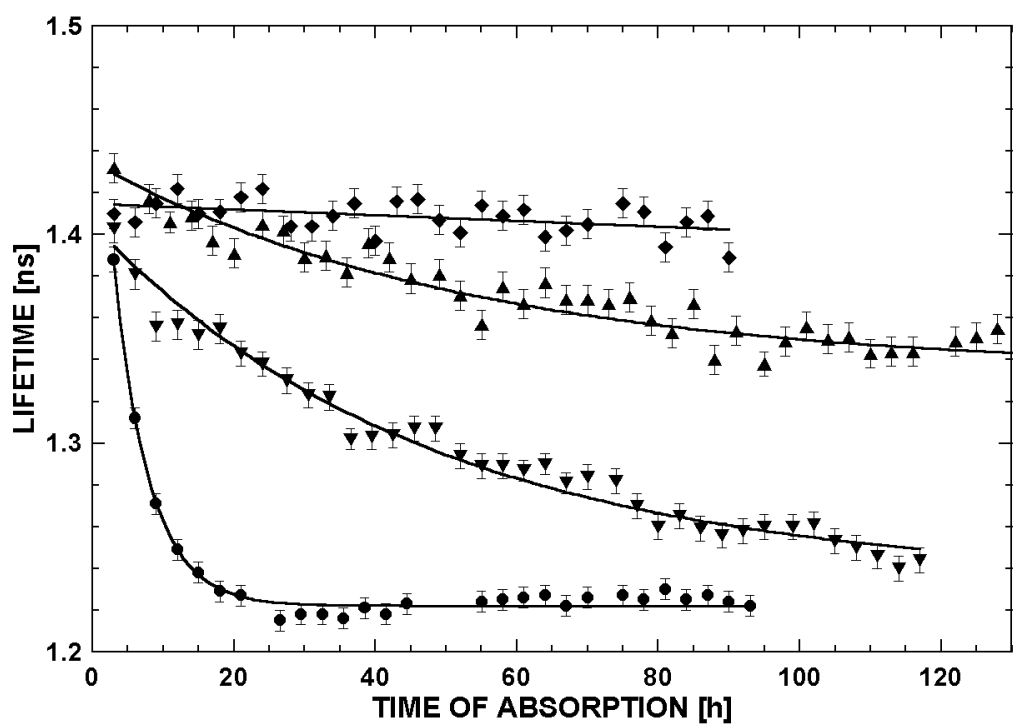

Fig. 3. Ortho-positronium lifetime as a function of vapour exposure time. Diamonds — dry air; dots - water; inverted triangles — hexane; triangles — hexanol.

Similar shortening of $\tau_{3}$ lifetime due to vapour absorption was observed by Ito et al. [1] in polyimide 6FDA-TMPD, i.e. cross-linked polymer in its glassy state, contrary to linear polymers in which vapour absorption leads to lifetime increase.

Lifetime shortening can be explained by a slight expansion of the free volume hole when a vapour molecule is located in it. Expansion of filled hole reduces the sizes of neighbouring voids. The o-Ps atom locates not in filled but in empty volume in the vicinity. Note that $\tau_{3}$ reduction from $1.4 \mathrm{~ns}$ to $1.22 \mathrm{~ns}$ means an average decrease in linear dimensions of the void by bare $0.022 \mathrm{~nm}$. The change of "natural" hole sizes is small comparing to changes in pore dimensions when MFR is produced in porous version (see Fig. 1). This interpretation of lifetime shortening has some drawbacks. The water molecule is the smallest of all used in this experiment, thus distortion of network should be smallest, too, as well as the lifetime shortening, contrary to the experimental results. However, in our experiment we observed the lifetime as a function of time, we have no information on the amount of absorbed vapour. If the amount of hexane (hexanol) absorbed is smaller comparing to water, one can suppose that majority of free volumes is still empty; the extent of distortion around the occupied one, averaged over whole polymer volume, can be small.

Pumping out vapour from the chamber volume leads to desorption of molecules from polymer matrix. The variation of o-Ps lifetime as a function of the pumping time is shown in Fig. 4. Water molecules were removed rapidly; already during the first 3 hours $\tau_{3}$ reached a value very close to that in MFR before absorption. The kinetic radius of water molecule is $\approx 0.15 \mathrm{~nm}[7]$, smaller than the average radius of voids in the polymer structure, thus water penetrates easily and also easily diffuses out. In the case of hexane and hexanol the behaviour of these 


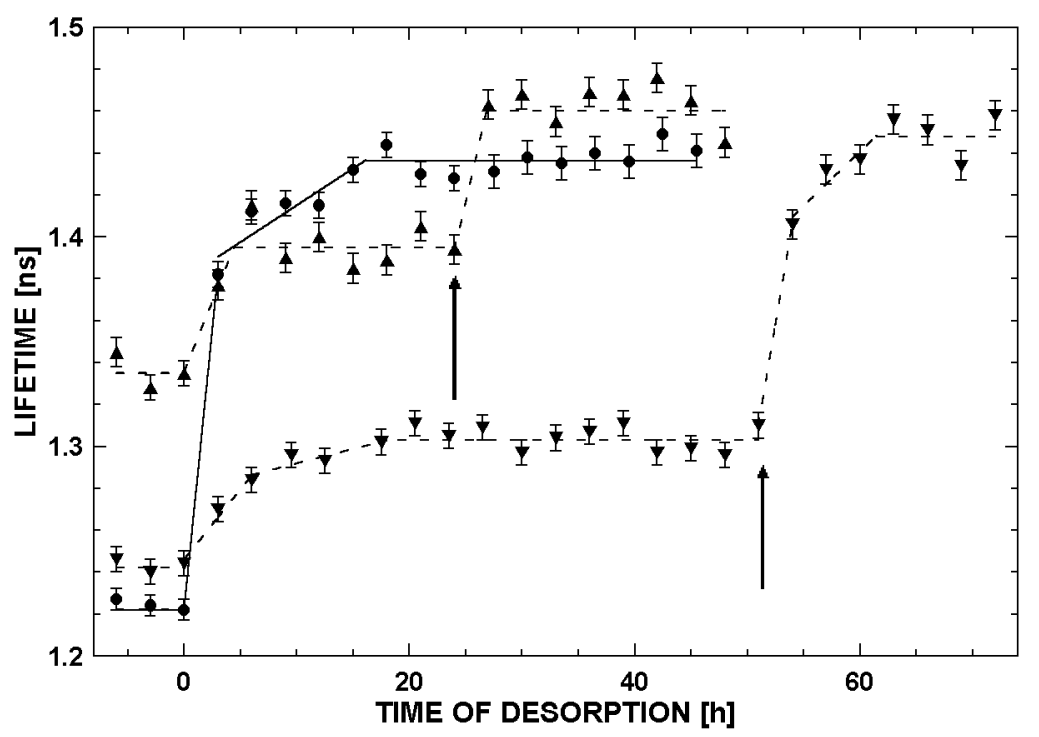

Fig. 4. Changes of o-Ps lifetime after evacuation of the sample chamber. Symbols are the same as in Fig. 3. Arrows indicate lifting of the sample temperature to $373 \mathrm{~K}$.

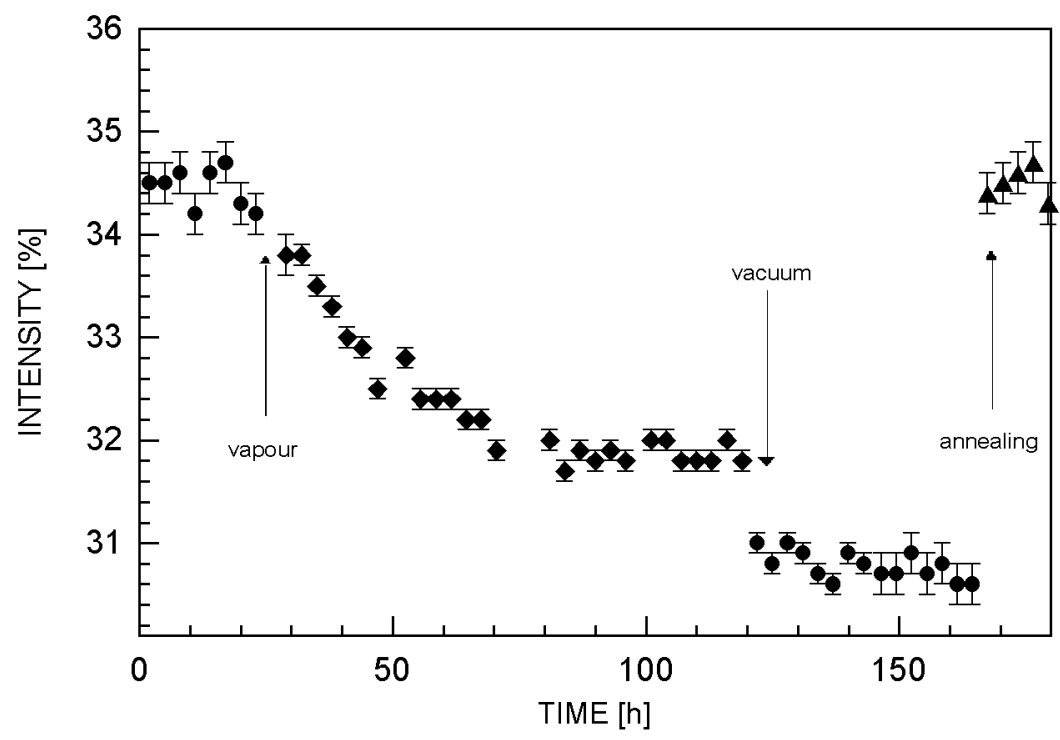

Fig. 5. Changes of long-lived component intensity during absorption and desorption of water. Arrows indicate from left to right: beginning of exposition of annealed sample to water vapour, chamber evacuation, increase in sample temperature to $370 \mathrm{~K}$.

molecules is more complex: during the first hours only a part of absorbed molecules was removed from the matrix; the lifetime $\tau_{3}$, initially rising fast, stopped at the level evidently lower than before vapour exposure. It was necessary to increase the sample temperature to $373 \mathrm{~K}$ to restore the initial value of the lifetime (the final $\tau_{3}$ 
value shown in Fig. 4 is higher than before absorption due to higher temperature - compare $\tau_{3}(T)$ dependence in Fig. 2). In the case of water absorption another effect is observed in Ps formation probability. During absorption the intensity $I_{3}$ slightly decreases, but after evacuation of the chamber, when $\tau_{3}$ is fully restored, the intensity decreases further (Fig. 5) and heating the sample becomes necessary to get the initial value of $I_{3}$. This effect needs to be studied in detail, e.g. as a function of temperature.

\section{Conclusions}

Positronium lifetime is very sensitive to small changes in hole geometry, thus it can be useful in studying distortion of polymer network. The observed changes of the structural void dimensions are averaged over the whole sample volume. One can suppose that during vapour absorption only a fraction of voids having the size of several angströms is filled; therefore, the decrease in the average radius of empty voids by $10 \%$ can mean larger expansion of filled ones. Size modification of nanovoids in non-porous MFR is evidently smaller than swelling of pores in porous modification of the same medium.

The lifetime rise during absorption observed by Ito et al. in linear polymers seems to be the result of much easier distortion of chains than of the cross-linked network; the vapour molecule placed between the chains bends them on the length much larger than the molecule size, creating thereby a new free space in its vicinity.

\section{References}

[1] Y. Ito, V. Sanchez, R. Lopez, L.A. Fucugauchi, K. Tanaka, K. Okamoto, Bull. Chem. Soc. Jpn. 66, 727 (1993).

[2] Y. Ito, in: Structure and Properties of Glassy Polymers, Eds. M.R. Tant, A.J. Hill, ACS Symposium Series 710, American Chemical Society, Washington 1998, p. 334.

[3] T. Suzuki, Y. Oki, M. Numajiri, T. Miura, K. Kondo, Y. Shiomi, Y. Ito, Polymer 37, 3025 (1996).

[4] Y. Ito, A. Shimadzu, K. Ikeda, Acta Phys. Pol. A 95, 546 (1999).

[5] P. Kirkegaard, N.J. Pedersen, M. Eldrup, Ris $\varnothing$ Report M2740, Ris $\varnothing$ National Laboratory, Denmark 1989.

[6] M. Eldrup, D. Lightbody, J.N. Sherwood, Chem. Phys. 63, 51 (1981).

[7] C.L. Soles, F.T. Chang, D.W. Gidley, A.F. Yee, J. Polym. Sci. Part B: Polym. Phys. 38, 776 (2000). 